\title{
UTILIZACÇÃO DE UMA CURVA DE CRESCIMENTO INTRA-UTERINO CORRIGIDO PARA PESO E ALTURA MATERNOS
}

\author{
Arnaldo Augusto Franco de Siqueira* \\ Cyro Ciari Junior * \\ Pedro Augusto Marcondes de Almeida*
}

\begin{abstract}
RSPU-B/260
Siqueira, A.A.F de et al, - Utilização de uma curva de crescimento intra-uterino corrigido para peso e altura maternos. Rev. Saúde públ., S. Paulo. 9:215-20, 1975.

Resumo: A necessidade do diagnóstico da desnutrição fetal levou à construção de várias curvas de crescimento intra-uterino. As diferenças étnicas $e$ sócio-econômicas entre as populaçōes impedem a utilização, em nosso meio, de curvas elaboradas em paises desenvolvidos. A precariedade dos registros de pré-natal $e$ de peso ao nascer torna muito difícil a construção de uma curva desse tipo. Foi demonstrada a validade da utilização da curva de crescimento intra-uterino de Tanner e Thomson, uma vez que ela introduz um fator de correção para a altura da mulher e para o peso na 20." semana da gestação. Os resultados obtidos permitem concluir que é possivel e fácil a aplicação da curva de Tanner e Thomson à população de gestantes sadias.
\end{abstract}

Unitermos: Crescimento fetal. Crescimento intra-uterino. Desnutriça intra-uterina. Peso ao nascer.

\section{NTRODUCA O}

Reconhece-se atualmente, entre os recém-nascidos de baixo peso (crianças nascidas com $2.500 \mathrm{~g}$ e menos) qualquer que seja a idade gestacional, dois grandes grupos: os de peso adequado e os de peso insuficiente para a idade gestacional.

Os recém-nascidos do $2 .^{\circ}$ grupo são muito diferentes daqueles do primeiro grupo, pois enquanto aqueles podem ser considerados como unicamente prematuros com peso inferior a $2.500 \mathrm{~g}$, estes sofreram um processo de desnutrição intrauterina.
Aliás, a desnutrição intra-uterina é, nas regiôes altamente desenvolvidas (como os países escandinavos e na maior parte dos Estados Lnidos, por exemplo), a única forma de dessautrição ainda presente.

Winick * (1974), calcula que nos ELA nasçam anualmente 70.000 crianças, pesando $2.500 \mathrm{~g}$ e menos, com peso insuficiente para a idade gestacional, crianças essas que vão engrossar as estatísticas de morbidade e mortalidade, bem como estarão sujeitas, ao longo da vida, a manifestar comprometimento neurológico ou retardo de desenvolvimento neuro-psicomotor.

* Disciplina Higiene Materna do Departamento de Prática Médica em Saúde Pública da USP - Av. Dr. Arnaldo, 715 - São Paulo, SP - Brasil 
SIQLEIRA. A.A.F. de et al - Litilizacão de uma curva de crescimento intra-uterino corrigidu para peso e altura maternos. Rev. Saude píbl., S. Paulo, 9:215-20, 1975.

St a este quadro acrescentarmos o fato te que parte dos nascimentos de crianças com peso insuficiente pode ser evitada à custa de rima hoa atenção pré-natal. vemos que a identificação, dentro da população de recém-nascidos, daqueles re-ultantes de crescimento intra-uterino inadequado, é um importante dado diagnóstico. seja a nível individual. orientando a conduta no herçário. seja a nível populacional. definindo necessidades programáticas.

Com a finalidade de identificar os recém-nascidos de peso insuficiente para a idade gestacional têm sido propostas várias curvas. nos últimos anos.

Assim é que Lubchenko e col. ${ }^{*}$ construiram uma curva de crescimento intrauterino, trabalhando com recém-nascidos de mulheres caucásicas da área de Denver, Colorado. Do total de nascidos viros, com idade gestacional conhecida, foram subtraídos os portadores de condiçóes patológicas que afetavam o peso ao nascer (anencefalia, hidrocefalia, hidropisia fetal e diahetes maternal. Foram ainda retiradas crianças cujo peso ao nas(er era incompatível com a idade gestarional.

Segundo o mesmo autor, crianças situadas abaixo do $10.0^{\circ}$ percentil podem ser -onsideradas pequenas para a idade gestacional.

Quando Lubchenko e col." comparam seus resultados, verifica-se que, idade por idade. os recém-nascidos são mais leves que os referidos por outros autores, atribuindo-se à altitude em que vive grande parte das mulheres cujos filhos foram incluídos no estudo. pois sabe-se que a altitude influi negativamente no peso ao nascer.

Cruenwald:" além de apresentar uma curva de crescimento intra-uterino. faz um estudo crítico das curvas até então apresentadas, verificando variações amplas. desde a curva de Kaltreider, com os valores mais haixos. oltida de uma população negra de status sócio-econômico muito haixo. até a de Lindell. com os valores mais altos. de crianças suecas. O mesmo autor. numa tentativa de construir a curva "ideal" de crescimento intra-uterino. considera-o quase linear no 3." trimestre da gravidez. Vários fatore: contribuem para desviar a tendência da curva para haixo, como a gemelaridade. o fumo e o baixo nível sócio-econômico. Sintetizando, Gruenwald ${ }^{3}$ diz que a curra diverge da reta por limitações da linha de suprimento fetal (mãe e placenta).

O que fica evidente da revisão feita por Gruenwald" é o falo de que a utilidade de cada curva, pelas peculiaridades da população de que derivam os recémnascidos, fica restrita a essa população.

Para a elahoração de uma curva deste tipo em nosso meio, seriam necessários milhares de registros corretos de fichas de pré-natal de mulheres sadias, além do peso ao nascer de seus filhos. Esse material é de obtenção muito difícil, tanto que. em trabalho anterior, trabalhamos com cerca de 500 observaçóes desse tipo :

Talvez a maior dificuldade para o emprego de uma curva em um meio diferente do original derive da grande diferença no tamanho das mulheres que compôem cada grupo, tanto que Gruenwald ${ }^{*}$. associando os valores da curva de Lindell à grande estatura das mulheres suecas. declara "que grozam um alto padrão de vida e presumivelmente grande estatura há gerações".

Butler e Bonham ${ }^{1}$, analisando dados do British Perinatal Mortality Survey mostram como a sstatura da mãe influi positivamente no peso ao nascer, mesmo em mulheres de mesmo status sócio-econômico. Thomson e col. ", trabalhando com 52.004 nascimentos simples e legítimos ocorridos em Aberdeen, na Escócia, com registros completos e corretos de pré-natal e peso ao nascer, publicaram uma tábua de peso por idade gestacional, que leva em conta o número de gestaçôes e o sexo do recém-nascido, mas que introduziu uma inovação muito interessante, em relação às demais, qual seja uma correção. para o lamanho materno. 
SIQUEIRA, A.A.F. de et al - Utiliząão de uma curva de crescimento intra-uterino corrigido para peso e altura maternos. Rev. Saide pübl., S Paulo, 9:215-20, 1975.

Tanner "Thomson ", utilizando esses resultados. construiram curvas para gestaçōes de 32 a 42 semanas, levando em conta o peso $:$ a altura maternos.

As diferenças entre os pesos dos recémnarcidos escoceses de Thomson e associados e os dos suecos de Sterky. por cles analisados. são anuladas com a introdução do fator de correção do peso. uma vez que as mulheres suecas são e'm média $5 \mathrm{~cm}$ maiores que as de Aberdeen.

Thomson e col." concluem dizendo que os seus resultados são provavelmente válidos para qualquer sociedade ocidental desenvolvida de raça branca, mas que eles não têm meios para saber se poderiam ser usados num grupo étnico diferente ou sol condiçôes sociais diferentes.

Num trabalho anterior" mostramos as relações entre altura materna, idade ges- lacional. restrição alimentar. (2 o pesio do recém-nascido.

Neste trahalho pretende-se mostrar a validade da utilização da curva de Tanner * Thomson num grupo étnico diferentr. sol condiçôes sociais diferentes das verrificadas em therdeen.

\section{MATERLAL E MÉTODOS}

() material é o mésmo já descrito em trabalho anterior". Foi utilizada uma lábua de correção do peso de recém-nas(ido (Tanner "Thomson "), isto é. quanlidades que deveriam ser adicionadas ou stuhtraídas aos pesos dos recém-nascidos. de acordo com a altura da mãe e o seu peso na 20. semana da gravidez (p)eso real ou extrapoladol.

T A B E L A 1

Correçōes a serem somadas ou subtraídas do peso ao nascer, de acordo com a altura da mãe e o peso na 20 a semana da gravidez*

\begin{tabular}{|c|c|c|c|c|c|c|c|c|c|c|c|}
\hline \multirow{2}{*}{$\begin{array}{l}\text { Altura } \\
\text { materna } \\
(\mathrm{cm})\end{array}$} & \multicolumn{10}{|c|}{ Peso materno no meio da gravidez ( $\mathrm{Kg}$ ) } & \multirow{2}{*}{$\begin{array}{c}\text { Com } \\
\text { peso } \\
\text { desconhecido }\end{array}$} \\
\hline & 35 & 40 & 45 & 50 & 55 & 60 & 65 & 70 & 75 & 80 & \\
\hline 145 & +550 & +475 & +400 & +325 & +250 & +175 & +100 & +25 & - & - & +330 \\
\hline 150 & +465 & +390 & +315 & +240 & +165 & +90 & +15 & -60 & - & - & +200 \\
\hline 155 & - & +320 & +245 & +170 & +95 & +20 & -55 & -130 & -205 & -280 & +80 \\
\hline 160 & - & +260 & +185 & +110 & +35 & -40 & -155 & -190 & -265 & -340 & -40 \\
\hline 165 & - & +215 & +140 & +65 & -10 & -85 & -160 & -235 & -310 & -385 & -140 \\
\hline 170 & - & - & +110 & +35 & -40 & -115 & -190 & -265 & -340 & -415 & -220 \\
\hline 175 & - & - & - & +15 & -60 & -135 & -210 & -285 & -360 & -435 & -280 \\
\hline 180 & - & - & - & - & -70 & -145 & -220 & -295 & -370 & -445 & -320 \\
\hline $\begin{array}{l}\text { Com altura } \\
\text { desconhecida }\end{array}$ & +510 & +390 & +275 & +165 & +60 & -40 & -135 & -225 & -310 & -395 & \\
\hline
\end{tabular}

* Extraida do trabalho de Tanner e Thomsons

Os pesos ao nascer corrigidos foram comparados com os resultados de Thomson e col. " e apresentados em percentis. Para melhor visualização dos resultados os mesmos foram dispostos sobre a curva de Tanner e Thomson"

Em virtude do pequeno número de casos em idades gestacionais menores apre- sentamos os resultados da $377^{\mathrm{a}}$ à $42 .^{\mathrm{a}}$ semana de gestação.

\section{RESULTADOS E DISCUSSAO}

Conforme se ohserva na Figura 10 peso ao nascer corrigido é praticamente o mesmo, independente da altura da mäe. Apenas no gruyo de mulheres de $1.60 \mathrm{~m}$ 
SIQUEIRA, A.A.F. de et al. - Utilização de uma curva de crescimento intra-uterino corrigido para peso e altura maternos. Rev. Saide públ., S. Paulo, 9:215-20, 1975.

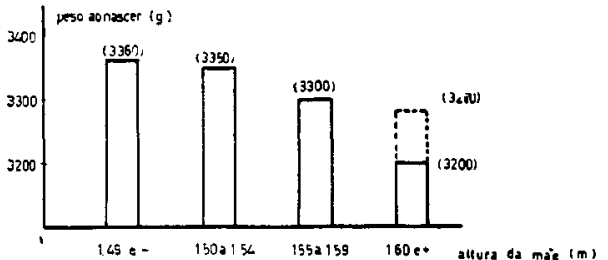

Obs.: a parte pontilhada mostra o aumento no peso ao nascer quando são retirados os casos de mulheres que sofreram restrição alimentar.

Fig. 1 - Peso ao nascer segundo a idade gestacional e corrigido para peso e altura da mãe. e mais houve uma discrepância maior. Se forem descartados os recém-nascidos cujas mães foram submetidas a restrição alimentar, no entanto, o peso médio nesse grupo atinge a $3.280 \mathrm{~g}$ (o peso médio dos - filhos de mulheres submetidas a restrição foi de $3.170 \mathrm{~g}$ ). Como em geral a restrição é introduzida no $30^{\circ}$ trimestre da gestação, esse efeito não pôde ser corrigido, pois o fator de correção é o peso na $20{ }^{2}$ semana.

A Figura 2 mostra os pesos médios ao nascer, corrigidos para o tamanho mater-

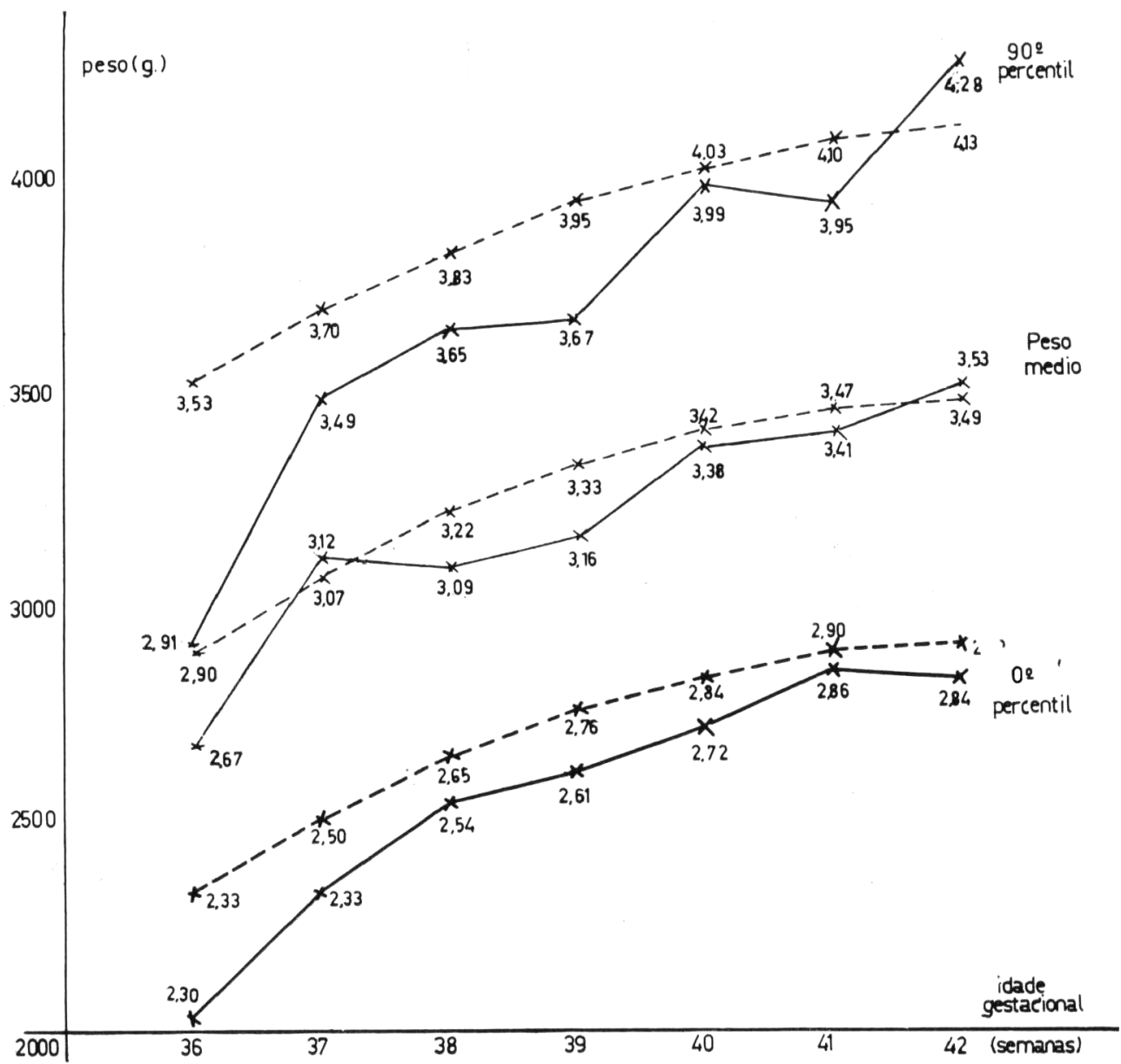

Flg. 2 - Comparação entre o peso corrigido do recém-nascido e os valores da curva de Tanner Thomson. 
SIQUEIRA, A.A.F. de et al. - Utilizacão de uma curva de crescimento intra-uterino corrigido para peso e altura maternos. Rev. Saúde públ., S. Paulo, 9:215-20, 1975.

no, de acordo com a idade gestacional, comparados aos pesos corrigidos da curva de Tanner e Thomson ". Foram também incluidos os pesos referentes ao $100^{\circ}$ e $900^{\circ}$ percentis.

Como se vê, os nossos resultados são semelhantes aos obtidos por Tanner $e$ Thomson ${ }^{5}$, porém em geral abaixo daqueles valores. Com a inclusão de um número maior de casos é possivel que parte dessa diferença desapareça.

Outro fator que poderá explicar, em parte, essa diferença foi o critério utilizado para calcular a idade gestacional, uma vez que Tanner e Thomson ${ }^{5}$ consideram apenas a idade em semanas completas, ou seja, toda criança nascida com idade gestacional de 37 semanas até 37 semanas e 6 dias, ao passo que nós consideramos as crianças de 37 semanas e 5 dias como de 38 semanas. Assim, parte de nossos casos que, de acordo com Tanner e Thomson" deveria figurar numa dada idade, é computada sempre na semana seguinte, o que faz diminuir o peso médio ao nascer.

Feitas essas ressalvas quanto au tamatho da amostra e ao cálculo da idade? gestacional, parece-nos perfeitamenle válida a utilização da curva de Tanner c Thomson em nosso meio, para a detecção dos recém-nascidos pequenos para a idade gestacional, através de um procedimento muito simples, qual seja fazer uma correção do pesn ao nascer segundo o tamanho materno antes de compará-lo com os valores da curva. Assim, os recém-nasridos situados abaixo do $10 .^{\circ}$ percentil podem ser considerados como de crescimento intra-uterino insuficiente.

Resta saber se a curva de Tanner e Thomson pode ser aplicada a qualquer grupo de gestantes sadias, pois as gestantes que freqüentam o Centro de Saúde Geraldo de Paula Souza da Faculdade de Saúde Pública da USP, como já foi visto em trabalhos anteriores, apresentam um status sócio-econômico acima da mé- dia da população de São Paulo. No entanto, como as mulheres são maiores quanto mais alto o nível sócio-econômico em que podem ser incluídas e esta curva corrige o efeito do tamanho da mulher sobre o peso do recém-nascido, a nossa impressão é a de que dará provavelmente resultados idênticos aos aqui apresentados.

Numa etapa posterior pretendemos avaliar a validade da utilização da curva em populações de gestantes de nível sócioeconômico mais baixo e mais alto que as aquí estudadas.

\section{CONCLUSOES}

1) Dadas as deficiências de registro clínico em pré-natal em nosso meio, é muilo difícil construir curvas fidedignas de crescimento intra-uterino.

2) As curvas de crescimento intrauterino que não levam em conta o tamanho da mulher normal, não podem ser utilizadas indiferentemente.

3) A introdução de um fator de correção para peso e altura da mulher normal permite a aplicação da curva de crescimento intra-uterino de Tanner e Thomson para populações diferentes da proposta.

4) Os resultados da aplicação de nossa casuística à curva de Tanner e Thomson permitem concluir pela sua validade.

5) Os dados de registro em pré-nalal c de peso ao nascer devem ser melhorados no sentido de que, utilizando curva adequada, possa ser feito o diagnóstico correto da desnutrição intra-uterina.

\section{A GRADECIMENTOS}

Ao Prof. Dr. Jair Licio Ferreira Santos e ao Sr. Fernão Dias de Lima pela confecção do programa para a computação dos resultados. 
SIQUEIRA, A.A.F. de et al - Utilização de uma curva de crescimento intra-uterino (orrigld" para peso e altura maternos Rev. Saite píbl., S. Paulo, 9:215-20, 1975.

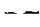

RSPU-B $/ 260$

Siqueira, A.A.F. de et al. - [On the use of an intrauterine growth curve corrected for maternal herght and werghtl. Rev. Saúde públ., S. Palulo. 9:215-20, 1975 .

Summary: Diagnostic requirements of fetal malnutrition brought into existence several intrauterne growth curves. The etnical and socioeconomical differences among pomulations do not allow us to use curves worked out in developed countries. The precarious records concerning pre-natal care and weight at birth hinder any attempt to build our own curves. In this paper the authors reveal the usefulness of Tanner and Thomson's intrauterine growth curve as it introduces a height and weight correction factor at the $20^{\text {h }}$ week of pregnancy. It is possible to conclude by the results obtained that Tanner and Thomson's curve can be very easily employed in healthy pregnant women at the S. Paulo University School of Public Health's Health Center "Geruldo de Paila Souza".

UNITERMS: Fetal growth. Intrauterine growth. Nutrition disorders, intrauterne. Birth weight.

\section{REFERENCIAS BIBLIOGRAFICAS}

1. BUTLER, N.R. \& BONHAM, D.G. Perinatal Mortality: the first report of the 1958 British Perinatal Mortality Survey. Edinburg, Livingstone. 1963.

2 CIARI JR., C. el al. - A relação entre o peso ao nascer. altura materna idade gestacional e restricão alimentar em gestantes normars. Rev. Saúde puibl., S. Paulo, 9:33-42, 1975.

3. GRUENWALD, P. - Growth of the human fetus. I. normal growth and its variation. Amer. Obstet Gymer, 94: $1112-9,1966$.

4. LUBCHENKO, L.O. et al - Intrauterine growth as estimated from liveborn hirth-weight data at 24 to 42 weeks of gestation. Pediatrics, 32:793-80n 1963 .

5. TANNER, J.M. \& THOMSON, A.M. Standards for birthweight at gestation periods from 32 to 42 weeks. allowing for maternal height Arch. Dis. Chithh. 45:566-9, 1970

6 THOMSON, A.M. et al - The assessment of fetal growth. J. Obstet. Gunaec. Brit. Cwith. 75:903-16, 1968.

7 WINICK, M. - Current status of malnutrition in the United States Amer. J. Trop. Med. Hyg. 23:752-55, 1974.

Recebido para publicasaio em 19-0.3-1975 Aprovado para publicaçó em o,-0/-1975 